\title{
A new view of the tree of life
}

\author{
Laura A. Hug1', Brett J. Baker², Karthik Anantharaman1, Christopher T. Brown ${ }^{3}$, Alexander J. Probst', \\ Cindy J. Castelle', Cristina N. Butterfield', Alex W. Hernsdorf ${ }^{3}$, Yuki Amano ${ }^{4}$, Kotaro Ise ${ }^{4}$, \\ Yohey Suzuki ${ }^{5}$, Natasha Dudek ${ }^{6}$, David A. Relman ${ }^{7,8}$, Kari M. Finstad ${ }^{9}$, Ronald Amundson ${ }^{9}$, \\ Brian C. Thomas ${ }^{1}$ and Jillian F. Banfield ${ }^{1,9 \star}$
}

\begin{abstract}
The tree of life is one of the most important organizing principles in biology'. Gene surveys suggest the existence of an enormous number of branches ${ }^{2}$, but even an approximation of the full scale of the tree has remained elusive. Recent depictions of the tree of life have focused either on the nature of deep evolutionary relationships ${ }^{3-5}$ or on the known, well-classified diversity of life with an emphasis on eukaryotes ${ }^{6}$. These approaches overlook the dramatic change in our understanding of life's diversity resulting from genomic sampling of previously unexamined environments. New methods to generate genome sequences illuminate the identity of organisms and their metabolic capacities, placing them in community and ecosystem contexts ${ }^{7,8}$. Here, we use new genomic data from over 1,000 uncultivated and little known organisms, together with published sequences, to infer a dramatically expanded version of the tree of life, with Bacteria, Archaea and Eukarya included. The depiction is both a global overview and a snapshot of the diversity within each major lineage. The results reveal the dominance of bacterial diversification and underline the importance of organisms lacking isolated representatives, with substantial evolution concentrated in a major radiation of such organisms. This tree highlights major lineages currently underrepresented in biogeochemical models and identifies radiations that are probably important for future evolutionary analyses.
\end{abstract}

Early approaches to describe the tree of life distinguished organisms based on their physical characteristics and metabolic features. Molecular methods dramatically broadened the diversity that could be included in the tree because they circumvented the need for direct observation and experimentation by relying on sequenced genes as markers for lineages. Gene surveys, typically using the small subunit ribosomal RNA (SSU rRNA) gene, provided a remarkable and novel view of the biological world ${ }^{1,9,10}$, but questions about the structure and extent of diversity remain. Organisms from novel lineages have eluded surveys, because many are invisible to these methods due to sequence divergence relative to the primers commonly used for gene amplification $^{7,11}$. Furthermore, unusual sequences, including those with unexpected insertions, may be discarded as artefacts ${ }^{7}$.

Whole genome reconstruction was first accomplished in 1995 (ref. 12), with a near-exponential increase in the number of draft genomes reported each subsequent year. There are 30,437 genomes from all three domains of life-Bacteria, Archaea and Eukaryawhich are currently available in the Joint Genome Institute's Integrated Microbial Genomes database (accessed 24 September 2015).
Contributing to this expansion in genome numbers are single cell genomics $^{13}$ and metagenomics studies. Metagenomics is a shotgun sequencing-based method in which DNA isolated directly from the environment is sequenced, and the reconstructed genome fragments are assigned to draft genomes ${ }^{14}$. New bioinformatics methods yield complete and near-complete genome sequences, without a reliance on cultivation or reference genomes ${ }^{7,15}$. These genome- (rather than gene) based approaches provide information about metabolic potential and a variety of phylogenetically informative sequences that can be used to classify organisms ${ }^{16}$. Here, we have constructed a tree of life by making use of genomes from public databases and 1,011 newly reconstructed genomes that we recovered from a variety of environments (see Methods).

To render this tree of life, we aligned and concatenated a set of 16 ribosomal protein sequences from each organism. This approach yields a higher-resolution tree than is obtained from a single gene, such as the widely used $16 \mathrm{~S}$ rRNA gene ${ }^{16}$. The use of ribosomal proteins avoids artefacts that would arise from phylogenies constructed using genes with unrelated functions and subject to different evolutionary processes. Another important advantage of the chosen ribosomal proteins is that they tend to be syntenic and co-located in a small genomic region in Bacteria and Archaea, reducing binning errors that could substantially perturb the geometry of the tree. Included in this tree is one representative per genus for all genera for which high-quality draft and complete genomes exist (3,083 organisms in total).

Despite the methodological challenges, we have included representatives of all three domains of life. Our primary focus relates to the status of Bacteria and Archaea, as these organisms have been most difficult to profile using macroscopic approaches, and substantial progress has been made recently with acquisition of new genome sequences ${ }^{7,8,13}$. The placement of Eukarya relative to Bacteria and Archaea is controversial ${ }^{1,4,5,17,18}$. Eukaryotes are believed to be evolutionary chimaeras that arose via endosymbiotic fusion, probably involving bacterial and archaeal cells ${ }^{19}$. Here, we do not attempt to confidently resolve the placement of the Eukarya. We position them using sequences of a subset of their nuclear-encoded ribosomal proteins, an approach that classifies them based on the inheritance of their information systems as opposed to lipid or other cellular structures ${ }^{5}$.

Figure 1 presents a new view of the tree of life. This is one of a relatively small number of three-domain trees constructed from molecular information so far, and the first comprehensive tree to

'Department of Earth and Planetary Science, UC Berkeley, Berkeley, California 94720, USA. ²Department of Marine Science, University of Texas Austin, Port Aransas, Texas 78373, USA. ${ }^{3}$ Department of Plant and Microbial Biology, UC Berkeley, Berkeley, California 94720, USA. ${ }^{4}$ Sector of Decommissioning and Radioactive Wastes Management, Japan Atomic Energy Agency, Ibaraki 319-1184, Japan. ${ }^{5}$ Graduate School of Science, The University of Tokyo, Tokyo 113-8654, Japan. ${ }^{6}$ Department of Ecology and Evolutionary Biology, UC Santa Cruz, Santa Cruz, California 95064, USA. 'Departments of Medicine and of Microbiology and Immunology, Stanford University, Stanford, California 94305, USA. ${ }^{8}$ Veterans Affairs Palo Alto Health Care System, Palo Alto, California 94304, USA. ${ }^{\circ}$ Department of Environmental Science, Policy, and Management, UC Berkeley, Berkeley, California 94720, USA. †Present address: Department of Biology, University of Waterloo, Waterloo, Ontario N2L 3G1, Canada. *e-mail: jbanfield@berkeley.edu 


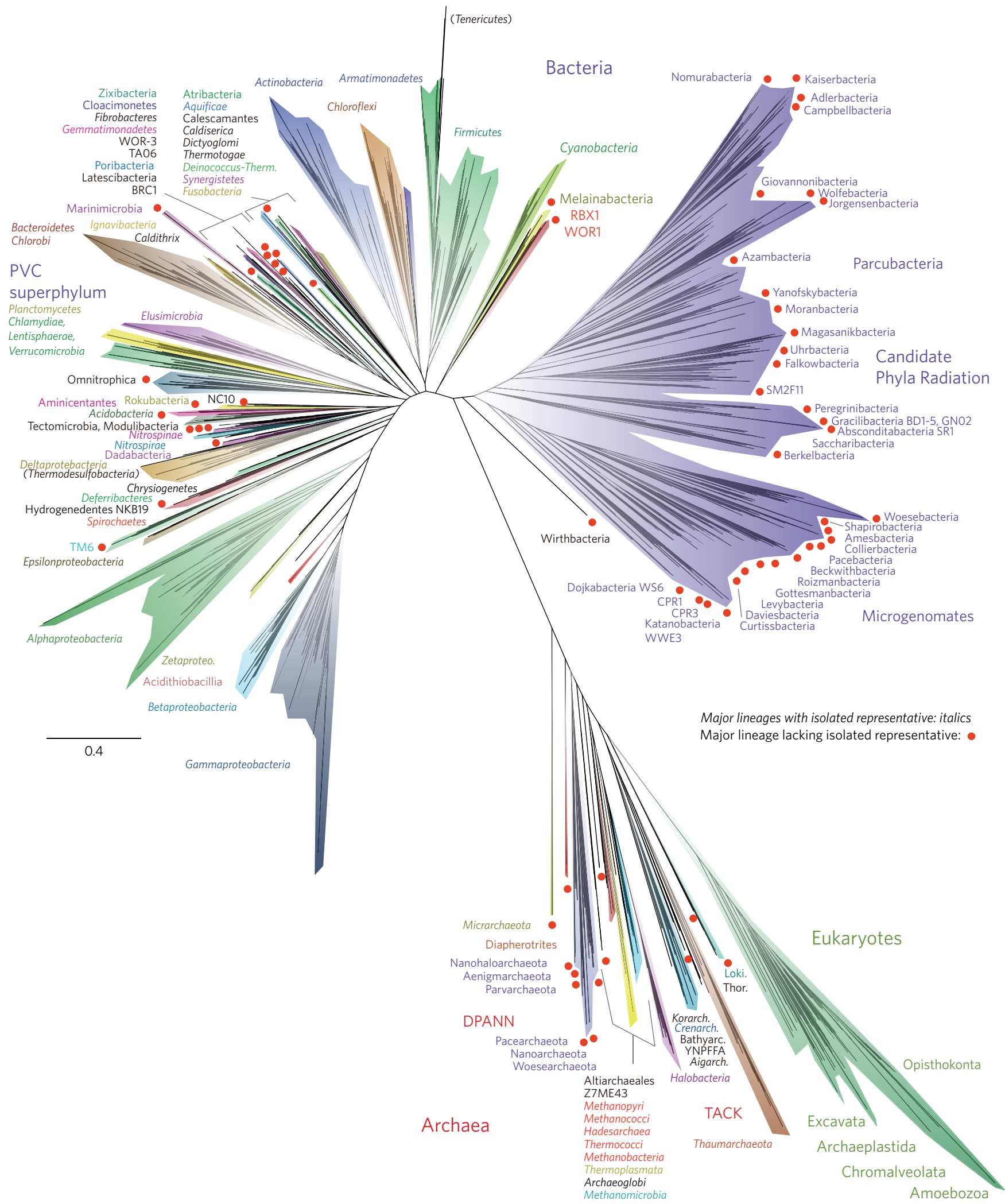

Figure 1 | A current view of the tree of life, encompassing the total diversity represented by sequenced genomes. The tree includes 92 named bacterial phyla, 26 archaeal phyla and all five of the Eukaryotic supergroups. Major lineages are assigned arbitrary colours and named, with well-characterized lineage names, in italics. Lineages lacking an isolated representative are highlighted with non-italicized names and red dots. For details on taxon sampling and tree inference, see Methods. The names Tenericutes and Thermodesulfobacteria are bracketed to indicate that these lineages branch within the Firmicutes and the Deltaproteobacteria, respectively. Eukaryotic supergroups are noted, but not otherwise delineated due to the low resolution of these lineages. The CPR phyla are assigned a single colour as they are composed entirely of organisms without isolated representatives, and are still in the process of definition at lower taxonomic levels. The complete ribosomal protein tree is available in rectangular format with full bootstrap values as Supplementary Fig. 1 and in Newick format in Supplementary Dataset 2. 


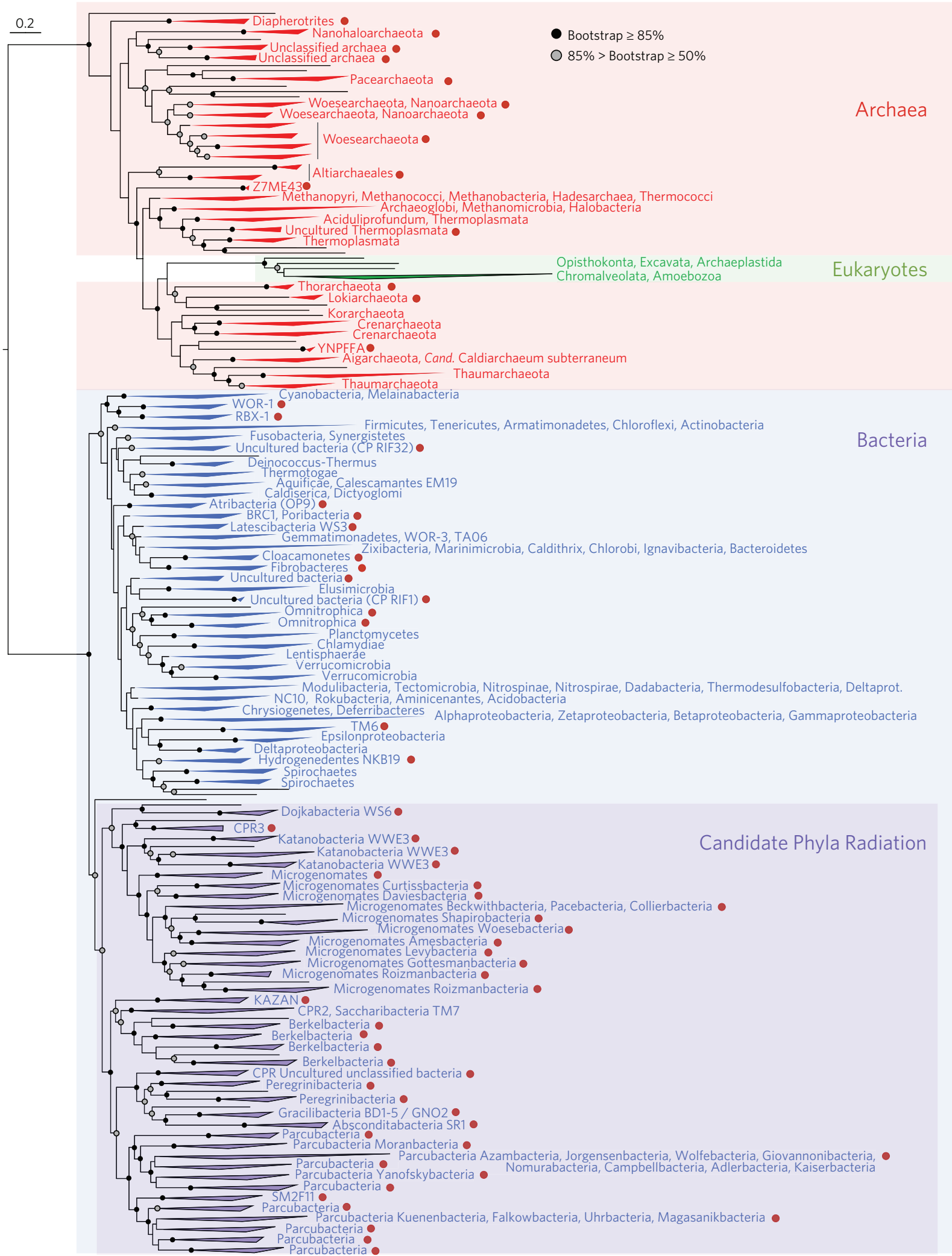

Figure 2 | A reformatted view of the tree in Fig. 1 in which each major lineage represents the same amount of evolutionary distance. The threshold for groups (coloured wedges) was an average branch length of $<0.65$ substitutions per site. Notably, some well-accepted phyla become single groups and others are split into multiple distinct groups. We undertook this analysis to provide perspective on the structure of the tree, and do not propose the resulting groups to have special taxonomic status. The massive scale of diversity in the CPR and the large fraction of major lineages that lack isolated representatives (red dots) are apparent from this analysis. Bootstrap support values are indicated by circles on nodes-black for support of $85 \%$ and above, grey for support from 50 to $84 \%$. The complete ribosomal protein tree is available in rectangular format with full bootstrap values as Supplementary Fig. 1 and in Newick format in Supplementary Dataset 2. 
be published since the development of genome-resolved metagenomics. We highlight all major lineages with genomic representation, most of which are phylum-level branches (see Supplementary Fig. 1 for full bootstrap support values). However, we separately identify the Classes of the Proteobacteria, because the phylum is not monophyletic (for example, the Deltaproteobacteria branch away from the other Proteobacteria, as previously reported ${ }^{2,20}$ ).

The tree in Fig. 1 recapitulates expected organism groupings at most taxonomic levels and is largely congruent with the tree calculated using traditional SSU rRNA gene sequence information (Supplementary Fig. 2). The support values for taxonomic groups are strong at the Species through Class levels $(>85 \%)$, with moderateto-strong support for Phyla ( $>75 \%$ in most cases), but the branching order of the deepest branches cannot be confidently resolved (Supplementary Fig. 1). The lower support for deep branch placements is a consequence of our prioritization of taxon sampling over number of genes used for tree construction. As proposed recently, the Eukarya, a group that includes protists, fungi, plants and animals, branches within the Archaea, specifically within the TACK superphylum ${ }^{21}$ and sibling to the Lokiarchaeota ${ }^{22}$. Interestingly, this placement is not evident in the SSU rRNA tree, which has the three-domain topology proposed by Woese and coworkers in $1990^{1}$ (Supplementary Fig. 2). The two-domain Eocyte tree and the three-domain tree are competing hypotheses for the origin of Eukarya ${ }^{5}$; further analyses to resolve these and other deep relationships will be strengthened with the availability of genomes for a greater diversity of organisms. Important advantages of the ribosomal protein tree compared with the SSU rRNA gene tree are that it includes organisms with incomplete or unavailable SSU rRNA gene sequences and more strongly resolves the deeper radiations. Ribosomal proteins have been shown to contain compositional biases across the three domains, driven by thermophilic, mesophilic and halophilic lifestyles as well as by a primitive genetic $\operatorname{code}^{23}$. Continued expansion of the number of genome sequences for non-extremophile Archaea, such as the DPANN lineages ${ }^{8,13}$, may allow clarification of these compositional biases.

A striking feature of this tree is the large number of major lineages without isolated representatives (red dots in Fig. 1). Many of these lineages are clustered together into discrete regions of the tree. Of particular note is the Candidate Phyla Radiation (CPR $)^{7}$, highlighted in purple in Fig. 1. Based on information available from hundreds of genomes from genome-resolved metagenomics and single-cell genomics methods to date, all members have relatively small genomes and most have somewhat (if not highly) restricted metabolic capacities ${ }^{7,13,24}$. Many are inferred (and some have been shown) to be symbionts ${ }^{7,25,26}$. Thus far, all cells lack complete citric acid cycles and respiratory chains and most have limited or no ability to synthesize nucleotides and amino acids. It remains unclear whether these reduced metabolisms are a consequence of superphylum-wide loss of capacities or if these are inherited characteristics that hint at an early metabolic platform for life. If inherited, then adoption of symbiotic lifestyles may have been a later innovation by these organisms once more complex organisms appeared.

Figure 2 presents another perspective, where the major lineages of the tree are defined using evolutionary distance, so that the main groups become apparent without bias arising from historical naming conventions. This depiction uses the same inferred tree as in Fig. 1, but with groups defined on the basis of average branch length to the leaf taxa. We chose an average branch length that best recapitulated the current taxonomy (smaller values fragmented many currently accepted phyla and larger values collapsed accepted phyla into very few lineages, see Methods). Evident in Fig. 2 is the enormous extent of evolution that has occurred within the CPR. The diversity within the CPR could be a result of the early emergence of this group and/or a consequence of rapid evolution related to symbiotic lifestyles. The CPR is early-emerging on the ribosomal protein tree (Fig. 1), but not in the SSU rRNA tree (Supplementary Fig. 2). Regardless of branching order, the CPR, in combination with other lineages that lack isolated representatives (red dots in Fig. 2), clearly comprises the majority of life's current diversity.

Domain Bacteria includes more major lineages of organisms than the other Domains. We do not attribute the smaller scope of the Archaea relative to Bacteria to sampling bias because metagenomics and single-cell genomics methods detect members of both domains equally well. Consistent with this view, Archaea are less prominent and less diverse in many ecosystems (for example, seawater $^{27}$, hydrothermal vents ${ }^{28}$, the terrestrial subsurface ${ }^{15}$ and human-associated microbiomes ${ }^{29}$ ). The lower apparent phylogenetic diversity of Eukarya is fully expected, based on their comparatively recent evolution.

The tree of life as we know it has dramatically expanded due to new genomic sampling of previously enigmatic or unknown microbial lineages. This depiction of the tree captures the current genomic sampling of life, illustrating the progress that has been made in the last two decades following the first published genome. What emerges from analysis of this tree is the depth of evolutionary history that is contained within the Bacteria, in part due to the CPR, which appears to subdivide the domain. Most importantly, the analysis highlights the large fraction of diversity that is currently only accessible via cultivation-independent genome-resolved approaches.

\section{Methods}

A data set comprehensively covering the three domains of life was generated using publicly available genomes from the Joint Genome Institute's IMG-M database (img. jgi.doe.gov), a previously developed data set of eukaryotic genome information ${ }^{30}$, previously published genomes derived from metagenomic data sets ${ }^{7,8,31,32}$ and newly reconstructed genomes from current metagenome projects (see Supplementary Table 1 for NCBI accession numbers). From IMG-M, genomes were sampled such that a single representative for each defined genus was selected. For phyla and candidate phyla lacking full taxonomic definition, every member of the phylum was initially included. Subsequently, these radiations were sampled to an approximate genus level of divergence based on comparison with taxonomically described phyla, thus removing strain- and species-level overlaps. Finally, initial tree reconstructions identified aberrant long-branch attraction effects placing the Microsporidia, a group of parasitic fungi, with the Korarchaeota. The Microsporidia are known to contribute long branch attraction artefacts confounding placement of the Eukarya ${ }^{33}$ and were subsequently removed from the analysis.

This study includes 1,011 organisms from lineages for which genomes were not previously available. The organisms were present in samples collected from a shallow aquifer system, a deep subsurface research site in Japan, a salt crust in the Atacama Desert, grassland meadow soil in northern California, a $\mathrm{CO}_{2}$-rich geyser system, and two dolphin mouths. Genomes were reconstructed from metagenomes as described previously ${ }^{7}$. Genomes were only included if they were estimated to be $>70 \%$ complete based on presence/absence of a suite of 51 single copy genes for Bacteria and 38 single copy genes for Archaea. Genomes were additionally required to have consistent nucleotide composition and coverage across scaffolds, as determined using the ggkbase binning software (ggkbase.berkeley.edu), and to show consistent placement across both SSU rRNA and concatenated ribosomal protein phylogenies. This contributed marker gene information for 1,011 newly sampled organisms, whose genomes were reconstructed for metabolic analyses to be published separately.

The concatenated ribosomal protein alignment was constructed as described previously ${ }^{16}$. In brief, the 16 ribosomal protein data sets (ribosomal proteins L2, L3, L4, L5, L6, L14, L16, L18, L22, L24, S3, S8, S10, S17 and S19) were aligned independently using MUSCLE v. 3.8.31 (ref. 34). Alignments were trimmed to remove ambiguously aligned $\mathrm{C}$ and $\mathrm{N}$ termini as well as columns composed of more than $95 \%$ gaps. Taxa were removed if their available sequence data represented less than $50 \%$ of the expected alignment columns ( $90 \%$ of taxa had more than $80 \%$ of the expected alignment columns). The 16 alignments were concatenated, forming a final alignment comprising 3,083 genomes and 2,596 amino-acid positions. A maximum likelihood tree was constructed using RAxML v. 8.1.24 (ref. 35), as implemented on the CIPRES web server ${ }^{36}$, under the LG plus gamma model of evolution (PROTGAMMALG in the RAxML model section), and with the number of bootstraps automatically determined (MRE-based bootstopping criterion). A total of 156 bootstrap replicates were conducted under the rapid bootstrapping algorithm, with 100 sampled to generate proportional support values. The full tree inference required 3,840 computational hours on the CIPRES supercomputer. 
To construct Fig. 2, we collapsed branches based on an average branch length criterion. Average branch length calculations were implemented in the Interactive Tree of Life online interface ${ }^{37}$ using the formula:

Average branch length=mean([root distance to tip] $-[$ root distance to node] $)$ for all tips connecting to a node.

We tested values between 0.25 and 0.75 at 0.05 intervals, and selected a final threshold of $<0.65$ based on generation of a similar number of major lineages as compared to the taxonomy-guided clustering view in Fig. 1. The taxonomy view identified 26 archaeal and 74 bacterial phylum-level lineages (counting the Microgenomates and Parcubacteria as single phyla each), whereas an average branch length of $<0.65$ resulted in 28 archaeal and 76 bacterial clades.

For a companion SSU rRNA tree, an alignment was generated from all SSU rRNA genes available from the genomes of the organisms included in the ribosomal protein data set. For organisms with multiple SSU rRNA genes, one representative gene was kept for the analysis, selected randomly. As genome sampling was confined to the genus level, we do not anticipate this selection process will have any impact on the resultant tree. All SSU rRNA genes longer than $600 \mathrm{bp}$ were aligned using the SINA alignment algorithm through the SILVA web interface ${ }^{38,39}$. The full alignment was stripped of columns containing $95 \%$ or more gaps, generating a final alignment containing 1,871 taxa and 1,947 alignment positions. A maximum likelihood tree was inferred as described for the concatenated ribosomal protein trees, with RAxML run using the GTRCAT model of evolution. The RAxML inference included the calculation of 300 bootstrap iterations (extended majority rules-based bootstopping criterion), with 100 randomly sampled to determine support values.

To test the effect of site selection stringency on the inferred phylogenies, we stripped the alignments of columns containing up to $50 \%$ gaps (compared with the original trimming of $95 \%$ gaps). For the ribosomal protein alignment, this resulted in a $14 \%$ reduction in alignment length (to 2,232 positions) and a $44.6 \%$ reduction in computational time $(\sim 2,100 \mathrm{~h})$. For the SSU rRNA gene alignment, stripping columns with $50 \%$ or greater gaps reduced the alignment by $24 \%$ (to 1,489 positions) and the computation time by $28 \%$. In both cases, the topology of the tree with the best likelihood was not changed significantly. The ribosomal protein resolved a twodomain tree with the Eukarya sibling to the Lokiarcheaota, while the SSU rRNA tree depicts a three-domain tree. The position of the CPR as deep-branching on the ribosomal protein tree and within the Bacteria on the SSU rRNA tree was also consistent. The alignments and inferred trees under the more stringent gap stripping are available upon request.

Nomenclature. We have included names for two lineages for which we have previously published complete genomes ${ }^{40}$. At the time of submission of the paper describing these genomes ${ }^{40}$, the reviewer community was not uniformly open to naming lineages of uncultivated organisms based on such information. Given that this practice is now widely used, we re-propose the names for these phyla. Specifically, for WWE3 we suggest the name Katanobacteria from the Hebrew 'katan', which means 'small', and for SR1 we suggest the name Absconditabacteria from the Latin 'Abscondo' meaning 'hidden', as in 'shrouded'.

Accession codes. NCBI and/or JGI IMG accession numbers for all genomes used in this study are listed in Supplementary Table 1. Additional ribosomal protein gene and 16S rRNA gene sequences used in this study have been deposited in Genbank under accession numbers KU868081-KU869521. The concatenated ribosomal protein and SSU rRNA alignments used for tree reconstruction are included as separate files in the Supplementary Information.

\section{Received 25 January 2016; accepted 10 March 2016; published 11 April 2016}

\section{References}

1. Woese, C. R., Kandler, O. \& Wheelis, M. L. Towards a natural system of organisms: proposal for the domains Archaea, Bacteria, and Eucarya. Proc. Natl Acad. Sci. USA 87, 4576-4579 (1990).

2. Yarza, P. et al. Uniting the classification of cultured and uncultured bacteria and archaea using $16 \mathrm{~S}$ rRNA gene sequences. Nature Rev. Microbiol. 12, 635-645 (2014)

3. Gouy, R., Baurain, D. \& Philippe, H. Rooting the tree of life: the phylogenetic jury is still out. Phil. Trans. R. Soc. Lond. B Biol. Sci. 370, 20140329 (2015).

4. Pace, N. R. Time for a change. Nature 441, 289 (2006).

5. Williams, T. A., Foster, P. G., Cox, C. J. \& Embley, T. M. An archaeal origin of eukaryotes supports only two primary domains of life. Nature 504, 231-236 (2013)

6. Hinchliff, C. E. et al. Synthesis of phylogeny and taxonomy into a comprehensive tree of life. Proc. Natl Acad. Sci. USA 112, 12764-12769 (2015).

7. Brown, C. T. et al. Unusual biology across a group comprising more than $15 \%$ of domain Bacteria. Nature 523, 208-211 (2015)

8. Castelle, C. J. et al. Genomic expansion of domain archaea highlights roles for organisms from new phyla in anaerobic carbon cycling. Curr. Biol. 25, 690-701 (2015).
9. Pace, N. R. Mapping the tree of life: progress and prospects. Microbiol. Mol. Biol. Rev. 73, 565-576 (2009).

10. Lane, D. J. et al. Rapid determination of $16 \mathrm{~S}$ ribosomal RNA sequences for phylogenetic analyses. Proc. Natl Acad. Sci. USA 82, 6955-6959 (1985).

11. Klindworth, A. et al. Evaluation of general $16 \mathrm{~S}$ ribosomal RNA gene PCR primers for classical and next-generation sequencing-based diversity studies. Nucleic Acids Res. 41, e1 (2013).

12. Fleischmann, R. et al. Whole-genome random sequencing and assembly of Haemophilus influenzae Rd. Science 269, 496-512 (1995).

13. Rinke, C. et al. Insights into the phylogeny and coding potential of microbial dark matter. Nature 499, 431-437 (2013).

14. Dick, G. J. et al. Community-wide analysis of microbial genome sequence signatures. Genome Biol. 10, R85 (2009).

15. Castelle, C. J. et al. Extraordinary phylogenetic diversity and metabolic versatility in aquifer sediment. Nature Commun. 4, 2120 (2013).

16. Hug, L. A. et al. Community genomic analyses constrain the distribution of metabolic traits across the Chloroflexi phylum and indicate roles in sediment carbon cycling. Microbiome 1, 22 (2013).

17. Cox, C. J., Foster, P. G., Hirt, R. P., Harris, S. R. \& Embley, T. M. The archaebacterial origin of eukaryotes. Proc. Natl Acad. Sci. USA 105, 20356-20361 (2008)

18. Lake, J. A., Henderson, E., Oakes, M. \& Clark, M. W. Eocytes: a new ribosome structure indicates a kingdom with a close relationship to eukaryotes. Proc. Natl Acad. Sci. USA 81, 3786-3790 (1984).

19. Martin, W. F., Garg, S. \& Zimorski, V. Endosymbiotic theories for eukaryote origin. Phil. Trans. R. Soc. B Biol. Sci. 370, 20140330 (2015).

20. Garrity, G., Boone, D. R. \& Castenholtz, R. W. (eds) Bergey's Manual of Systematic Bacteriology. Volume One: The Archaea and the Deeply Branching and Phototrophic Bacteria 2nd edn (Springer, 2001).

21. Guy, L. \& Ettema, T. J. G. The archaeal 'TACK' superphylum and the origin of eukaryotes. Trends Microbiol. 19, 580-587 (2011).

22. Spang, A. et al. Complex archaea that bridge the gap between prokaryotes and eukaryotes. Nature 521, 173-179 (2015).

23. Fournier, G. P. \& Gogarten, J. P. Rooting the ribosomal tree of life. Mol. Biol. Evol. 27, 1792-1801 (2010).

24. Wrighton, K. C. et al. Fermentation, hydrogen, and sulfur metabolism in multiple uncultivated bacterial phyla. Science 337, 1661-1665 (2012).

25. He, X. et al. Cultivation of a human-associated TM7 phylotype reveals a reduced genome and epibiotic parasitic lifestyle. Proc. Natl Acad. Sci. USA 112, 244-249 (2015).

26. Nelson, W. C. \& Stegen, J. C. The reduced genomes of Parcubacteria (OD1) contain signatures of a symbiotic lifestyle. Front. Microbiol. 6, 713 (2015).

27. Lloyd, K. G., May, M. K., Kevorkian, R. T. \& Steen, A. D. Meta-analysis of quantification methods shows that archaea and bacteria have similar abundances in the subseafloor. Appl. Environ. Microbiol. 79, 7790-7799 (2013).

28. Sievert, S. M., Ziebis, W., Kuever, J. \& Sahm, K. Relative abundance of Archaea and Bacteria along a thermal gradient of a shallow-water hydrothermal vent quantified by rRNA slot-blot hybridization. Microbiology 146, 1287-1293 (2000).

29. Hoffmann, C. et al. Archaea and fungi of the human gut microbiome: correlations with diet and bacterial residents. PLoS ONE 8, e66019 (2013).

30. Brown, M. W. et al. Phylogenomics demonstrates that breviate flagellates are related to opisthokonts and apusomonads. Proc. Biol. Sci. 280, 20131755 (2013).

31. Hug, L. A. et al. Critical biogeochemical functions in the subsurface are associated with bacteria from new phyla and little studied lineages. Environ. Microbiol. 18, 159-173 (2016).

32. Baker, B. J., Lazar, C. S., Teske, A. P. \& Dick, G. J. Genomic resolution of linkages in carbon, nitrogen, and sulfur cycling among widespread estuary sediment bacteria. Microbiome 3, 14 (2015).

33. Inagaki, Y., Susko, E., Fast, N. M. \& Roger, A. J. Covarion shifts cause a longbranch attraction artifact that unites microsporidia and archaebacteria in EF1alpha phylogenies. Mol. Biol. Evol. 21, 1340-1349 (2004).

34. Edgar, R. C. MUSCLE: multiple sequence alignment with high accuracy and high throughput. Nucleic Acids Res. 32, 1792-1797 (2004).

35. Stamatakis, A. RAxML-VI-HPC: maximum likelihood-based phylogenetic analyses with thousands of taxa and mixed models. Bioinformatics 22, 2688-2690 (2006)

36. Miller, M., Pfeiffer, W. \& Schwartz, T. Creating the CIPRES Science Gateway for inference of large phylogenetic trees. Gatew. Comput. Environ. Work 1-8 (2010).

37. Letunic, I. \& Bork, P. Interactive Tree Of Life v2: online annotation and display of phylogenetic trees made easy. Nucleic Acids Res. 39, W475-W478 (2011).

38. Pruesse, E., Peplies, J. \& Glöckner, F. O. SINA: accurate high-throughput multiple sequence alignment of ribosomal RNA genes. Bioinformatics 28, 1823-1829 (2012)

39. Pruesse, E. et al. SILVA: a comprehensive online resource for quality checked and aligned ribosomal RNA sequence data compatible with ARB. Nucleic Acids Res. 35, 7188-7196 (2007) 
40. Kantor, R. S. et al. Small genomes and sparse metabolisms of sedimentassociated bacteria from four candidate phyla. MBio 4, e00708-13 (2013).

\section{Acknowledgements}

This research was largely supported by the Lawrence Berkeley National Laboratory (LBNL)

Genomes to Watershed Scientific Focus Area funded by the US Department of Energy (DOE),

Office of Science, Office of Biological and Environmental Research (OBER) under contract no. DE-AC02-05CH11231. Additional support was provided by LBNL EFRC award no. DEAC02-05CH11231, NASA NESSF grant no. 12-PLANET12R-0025 and NSF DEB grant no. 1406956, DOE OBER grant no. DOE-SC10010566, Office of Naval Research grants nos. N00014-07-1-0287, N00014-10-1-0233 and N00014-11-1-0918, and by the Thomas C. and Joan M. Merigan Endowment at Stanford University. In addition, funding was provided by the Ministry of Economy, Trade and Industry of Japan. The authors thank J. Eisen for comments, S. Venn-Watson, K. Carlin and E. Jensen (US Navy Marine Mammal Program) for dolphin samples, K.W. Seitz for sequence submission assistance, and the DOE Joint Genome Institute for generating the metagenome sequence via the Community Science Program.

\section{Author contributions}

L.A.H. and J.F.B. defined the research objective. L.A.H. generated data sets and conducted phylogenetic tree inferences. L.A.H., B.J.B. and J.F.B. conducted data analysis. L.A.H.,
B.J.B., K.A., C.T.B., A.J.P., C.J.C., C.N.B., A.W.H., Y.A., K.I., Y.S., N.D., D.A.R., K.M.F., R.A B.C.T. and J.F.B. contributed to metagenome binning and genome analysis. L.A.H. and J.F.B. wrote the manuscript with input from all authors. All authors read and approved the final manuscript.

\section{Additional information}

Supplementary information is available online. Reprints and permissions information is available online at www.nature.com/reprints. Correspondence and requests for materials should be addressed to J.F.B.

\section{Competing interests}

The authors declare no competing financial interests.

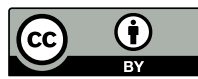

This work is licensed under a Creative Commons Attribution 4.0 International License. The images or other third party material in this article are included in the article's Creative Commons license, unless indicated otherwise in the credit line; if the material is not included under the Creative Commons license, users will need to obtain permission from the license holder to reproduce the material. To view a copy of this license, visit http://creativecommons.org/ licenses/by/4.0/ 